\title{
Observation of the Underscreened Kondo Effect in a Molecular Transistor
}

\author{
Nicolas Roch, ${ }^{1}$ Serge Florens, ${ }^{1}$ Theo A. Costi, ${ }^{2}$ Wolfgang Wernsdorfer, ${ }^{1}$ and Franck Balestro ${ }^{1}$ \\ ${ }^{1}$ Institut Néel, associé à l'UJF, CNRS, BP 166, 38042 Grenoble Cedex 9, France \\ ${ }^{2}$ Institut für Festkörperforschung, Forschungszentrum Jülich, 52425 Jülich, Germany
}

(Received 5 May 2009; published 3 November 2009)

\begin{abstract}
We present the first quantitative experimental evidence for the underscreened Kondo effect, an incomplete compensation of a quantized magnetic moment by conduction electrons, as originally proposed by Nozières and Blandin. The device consists of an even charge spin $S=1$ molecular quantum dot, obtained by electromigration of $\mathrm{C}_{60}$ molecules into gold nanogaps and operated in a dilution fridge. The persistence of logarithmic singularities in the low temperature conductance is demonstrated by a comparison to the fully screened configuration obtained in odd charge spin $S=1 / 2$ Coulomb diamonds. We also discover an extreme sensitivity of the underscreened Kondo resonance to the magnetic field that we confirm on the basis of numerical renormalization group calculations.
\end{abstract}

PACS numbers: 75.20.Hr, 71.10.Hf, 73.21.La, 73.63.-b

When a magnetic impurity is inserted in a piece of metal, its magnetic moment can be completely screened by the conduction electrons, owing to their quantized spin $1 / 2$. This general phenomenon, the Kondo effect, has been thoroughly studied in diluted magnetic alloys [1] and has attracted considerable attention in the more recent quantum dot systems [2]. Clearly, impurities carrying a spin $S$ greater that $1 / 2$ need to bind several electronic orbitals in order to fully quench their magnetism, and nature seems to conspire in always providing enough screening channels for that situation to occur in general [3]. Therefore, the possibility that screening may happen to be incomplete, as initially proposed on theoretical grounds by Nozières and Blandin [4], has remained elusive for almost 30 years despite the great experimental control that one can achieve with artificial quantum dot setups. The observation of the underscreened Kondo effect, in addition to its overscreened counterpart [5], is also especially appealing since it constitutes one of the simplest cases where standard Fermiliquid theory is violated $[6,7]$.

We demonstrate in this Letter that molecular quantum dots obtained through electromigration [8] are perfect candidates for achieving underscreened Kondo impurities. Indeed, point contact tunneling (single mode) and important left or right asymmetry of the transport electrodes ensure a large window of energies where a single screening channel is active. In addition, the Kondo phenomenon in molecules can set in already at several kelvins [9-12] thanks to relatively important charging energies, allowing a complete study of Kondo crossovers on a sufficient range of temperatures. Both conditions of single channel and large Kondo temperature are difficult to meet altogether in other quantum dot devices, where Kondo effects associated with higher spin states have been previously found but not yet investigated in detail [13-17]. We report here on the first observation of the anomalous logarithmic behavior in the temperature and bias voltage dependent conduc- tance in a spin $S=1$ quantum dot below the Kondo scale, as previously predicted for underscreened impurities $[4,6,7,18]$, and we successfully confront our results with quantitative numerical renormalization group (NRG) calculations. More strikingly, the experimental data demonstrate that underscreened impurities are extremely sensitive to the application of a magnetic field. In that case, and in contrast to fully screened moments, the Kondo resonance is split by a Zeeman energy much smaller than the Kondo temperature, reflecting the high degree of polarizability of a partially screened spin. This surprising finding is confirmed by new NRG calculations of the local density of states that we performed on the single-channel spin $S=1$ Kondo model in a magnetic field.

Our recent work [19] has shown that $\mathrm{C}_{60}$ quantum dots with two electrons on the molecule can be gate tuned via a quantum phase transition between a molecular singlet and a spin $S=1$ Kondo state. Interestingly, the central condition for this phase transition to occur, vindicated by these experimental findings [19], is the presence of a single screening channel in the accessible temperature range $[20,21]$. Since screening channels result from the overlap between the wave functions of the conduction electrons and those of the magnetic impurity orbitals [4], one understands that orbital quantum numbers must not be preserved in order for the underscreened Kondo effect to take place [see Fig. 1(a) discussing the situation of $n_{\mathrm{sc}}$ channels coupled to an impurity spin $S]$. We can describe our molecular transistor [19] by two orbital levels $(-1,+1)$ coupled to two metallic leads $(L, R)$ [see Fig. 1(b)], with the tunneling matrix [20]:

$$
\mathbf{t}=\left(\begin{array}{cc}
t_{L,+1} & t_{R,+1} \\
t_{L,-1} & t_{R,-1}
\end{array}\right) .
$$

A screening channel $\lambda=1,2$ is associated to each eigenvalue $t_{\lambda}$ of this matrix, from which antiferromagnetic Kondo couplings $J_{\lambda}=4\left|t_{\lambda}\right|^{2} / E_{\text {add }}$ result between the lo- 
a

\begin{tabular}{|l|l|}
\hline $2 \mathrm{~S}>\mathrm{n}_{\mathrm{SC}}$ & underscreened \\
\hline $2 \mathrm{~S}=\mathrm{n}_{\mathrm{SC}}$ & fully screened \\
\hline $2 \mathrm{~S}<\mathrm{n}_{\mathrm{SC}}$ & overscreened \\
\hline
\end{tabular}

b
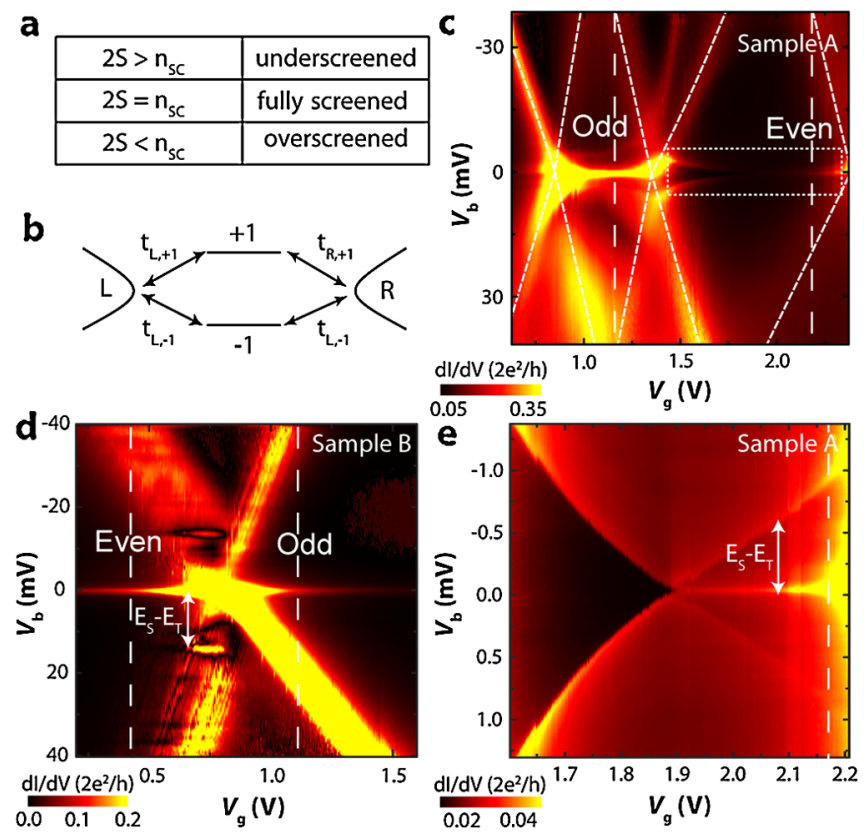

FIG. 1 (color online). (a) Summary of the different types of Kondo effects according to impurity spin $S$ and number of screening channels $n_{\mathrm{sc}}$. (b) Tunneling model of our single molecule transistor: two orbital levels couple to two leads. (c) Conductance map of sample $A$ recorded at $T=35 \mathrm{mK}$. Dashed lines are positioned at the gate voltages where more detailed studies where performed. (d) Conductance map for sample $B$. (e) Zoom inside the dotted rectangle defined in the even diamond of sample $A$. The white arrows represent the singlet-triplet splitting in both samples.

calized orbitals and the conduction electrons ( $E_{\text {add }}$ is a measure of the addition energy on the quantum dot). One then obtains two Kondo temperatures:

$$
k_{B} T_{K \lambda}=\sqrt{D J_{\lambda}} e^{-1 /\left(\rho J_{\lambda}\right)},
$$

where $D$ is the bandwidth of the metal and $\rho$ its the density of states. While it seems physically sound that at vanishing temperature a spin $S=1$ will be fully screened [20], moderate left or right asymmetries in the tunneling amplitudes (1) can lead to vastly different Kondo scales because of the exponential behavior in (2), as pointed out in [18]. This situation naturally occurs with electromigration, as the molecule tends to stay preferably closer to one of the electrodes so that, e.g., $t_{L, \pm 1} \gg t_{R, \pm 1}$. Figure 1 shows indeed that our conductance maxima, here shown for two different devices, are much lower than the quantum value $2 e^{2} / h$, with $e$ the electron charge and $h$ Planck's constant. It is then possible to have a large range of temperatures over which underscreened behavior prevails. A further crucial condition for the realization of the underscreened Kondo effect is the formation of a spin $S=1$ unit itself. This relies on a strong Hund's rule that makes the separation between singlet and triplet states much larger than the temperature. These high-energy singlet excitations [22] are clearly spectroscopically resolved in all of our measure- ments through cotunneling lines [see Figs. 1(d) and 1(e)]. Sample $A$ in Fig. 1(c) shows an odd charge Coulomb diamond with a pronounced Kondo ridge for gate voltages between $V_{g}=0.8 \mathrm{~V}$ and $1.35 \mathrm{~V}$ (parity was argued in [19]), so that the next diamond seen up to $V_{g}=2.3 \mathrm{~V}$ has an even number of electrons. Figure 1(d) represents the conductance map of sample $B$, where a single charge degeneracy point is observed, with Kondo ridges on both sides. We estimate a low limit for the addition energy $E_{\text {add }} \geq 200 \mathrm{meV}$. Figure 1(e) focuses inside the even diamond of sample $A$, where the singlet to triplet quantum phase transition occurs [19]. We can thus assess that our experiment was carried out in the temperature range $T_{K 1} \ll T \ll T_{K 2}$. Similar singlet excitations are visible on the left-hand side of sample $B$ [see Fig. 1(d)], thus allowing us to label this region even.

We now present the study of our two different devices [see Figs. 1(c) and 1(d)], both showing fully screened and underscreened Kondo anomalies. In order to maximize the Kondo temperature, gate voltages were chosen away from the center of the Coulomb diamonds while staying out of the mixed valence regime. In the fully screened Kondo effect, the conductance versus lowering the temperature has a logarithmic increase above the Kondo temperature and then saturates in a quadratic, Fermi-liquid-like fashion. On the other hand, the underscreened Kondo effect is expected to show two distinct logarithmic behaviors $[4,6,7,18]$ above and below the highest Kondo temperature $T_{K 2}$. Indeed, partially screened moments are known to act as ferromagnetic Kondo impurities [4], leading to a slow logarithmic scattering of the conduction electrons at low energy. This is best seen by plotting the derivative of conductance with respect to temperature as a function of inverse temperature, as suggested in [18]. Our data in the inset of Fig. 2(a), taken for the even diamond of sample $A$, clearly display two distinct $1 / T$ regimes. Another way to discriminate both Kondo effects is to perform a fit to NRG results $[3,23]$ of single-channel spin $S=1 / 2$ and $S=1$ Kondo models. The obvious qualitative differences, namely, the apparent divergence in $d G(T) / d T$ at low temperature $T$ for spin $S=1$ and the presence of a maximum for spin $S=1 / 2$, in addition to the quantitative agreement with the NRG predictions, are strong indications of two remarkably different Kondo states [see Figs. 2(a) and 2(b)]. This comparison to theory allows us to extract the associated Kondo temperatures $T_{K}$ and the conductance amplitude $G_{0}$ for each curve, using the scaling form

$$
G(T)=G_{0} f_{S}^{\mathrm{NRG}}\left(T / T_{K}\right)+G_{\mathrm{bg}},
$$

with $S$ the impurity spin and $G_{\mathrm{bg}}$ a constant conductance background [24]. We stopped our measurements around $T_{\text {base }}=100 \mathrm{mK}$ in order to ensure that saturation effects are not provoked by an uncontrolled electronic temperature. We also limited the ac excitation of the lock-in detection to stay in the limit $e V_{\text {ac }} \ll k_{B} T$. With these words of caution, we could not observe any saturation of 

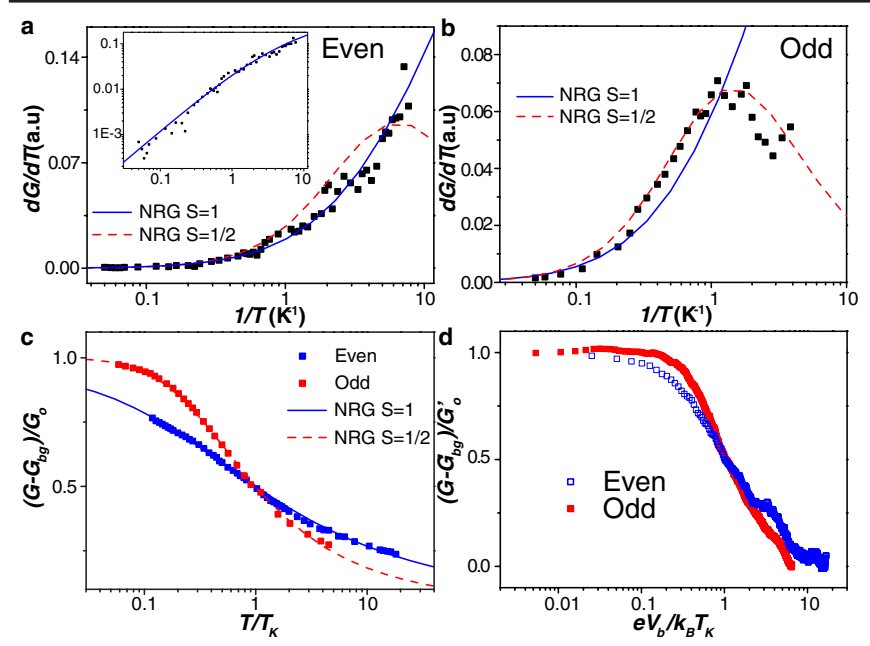

FIG. 2 (color online). (a) Derivative of the conductance versus temperature recorded at gate $V_{g}=2.17 \mathrm{~V}$ and bias $V_{b}=0 \mathrm{~V}$ in the even Coulomb diamond of sample $A$ (same data on a $\log$ scale in the inset, showing two distinct $1 / T$ behaviors). The best fit is obtained for an underscreened Kondo model (solid line) and gives $G_{0}=0.14$ (in units of $2 e^{2} / h$ ) and $T_{K}=1.1 \mathrm{~K}$. (b) Derivative of the conductance versus temperature recorded at $V_{g}=1.2 \mathrm{~V}$ and $V_{b}=0 \mathrm{~V}$ in the odd Coulomb diamond. The best fit is obtained for a fully screened Kondo model (dashed line) and gives $G_{0}=0.34$ and $T_{K}=4.4 \mathrm{~K}$. (c) Conductance data of (a) and (b) compared to the relevant NRG curves. The background was adjusted to follow the convention $G\left(T_{K}\right)=$ $G_{0} / 2$, giving $G_{\mathrm{bg}}=0.055$ in the fully screened case and $G_{\mathrm{bg}}=$ 0.022 in the underscreened case. (d) Differential conductance at the base temperature rescaled in universal form, with $G_{0}^{\prime}=$ $G\left(T_{\text {base }}\right)$ and $G_{\mathrm{bg}}^{\prime}$ such that the conductance is $G_{0}^{\prime} / 2$ at $e V_{b}=$ $k_{B} T_{K}$.

the conductance over two decades of temperature in the even diamond, contrary to what is witnessed in the fully screened case. Since the Kondo effect displays a universal behavior with a single scale $T_{K}$, this must be reflected by different universal curves between screened and underscreened situations, as evidenced by Fig. 2(c). The bias voltage dependence of the nonlinear conductance at the base temperature was also examined [25,26]. Although this study is more difficult to perform accurately, two relatively different curves are nevertheless obtained with slower voltage behavior for the spin $S=1$ molecules [see Fig. 2(d)]. We also note that a measure of $T_{K}$ can be roughly deduced from the half width at half maximum (HWHM) of the nonlinear conductance peak; this method was used to have an alternative determination of $T_{K}$ for each Kondo ridge in samples $A$ and $B$ after extraction of a background contribution (see Table I). The higher error bars for the bias method are due to the absence of reliable finite voltage predictions from theory; thus, $T_{K}$ depends sensitively on the estimated background. Both methods, relying on thermal or voltage smearing of the Kondo peak, nicely converge to comparable $T_{K}$ values for both types of Kondo effects.
TABLE I. Kondo temperatures for each sample in the even and odd diamonds, determined by using the methods of temperature, bias, or magnetic field, as described in the text. Sample $B$ was not stable enough to perform a detailed temperature study.

\begin{tabular}{lccc}
\hline \hline Sample/Method & Temperature & Bias & Magnetic Field \\
\hline$A$ (odd) & $4.4 \pm 0.3 \mathrm{~K}$ & $5.5 \pm 1.3 \mathrm{~K}$ & $4.8 \pm 0.3 \mathrm{~K}$ \\
$A$ (even) & $1.1 \pm 0.1 \mathrm{~K}$ & $1.9 \pm 0.5 \mathrm{~K}$ & $0.6 \pm 0.4 \mathrm{~K}$ \\
$B$ (odd) & & $4.4 \pm 0.8 \mathrm{~K}$ & $5.4 \pm 0.3 \mathrm{~K}$ \\
$B$ (even) & & $1.9 \pm 0.3 \mathrm{~K}$ & $0.2 \pm 0.1 \mathrm{~K}$ \\
\hline \hline
\end{tabular}

The evolution of the Kondo peak as a function of magnetic field, as seen in the bias spectrum of Figs. 3(a) and 3(b), displays even more dramatic effects. Previous experimental studies $[17,27,28]$ and theoretical calculations [29] on fully screened impurities have shown a Zeeman splitting of the Kondo resonance at a critical field $g \mu_{B} B_{c} \sim 0.5 k_{B} T_{K}$. Indeed, as seen in Table I, this estimate agrees with the $T_{K}$ determined by the temperature and bias dependence for the odd charge diamonds of both samples. In contrast, for the spin $S=1$ quantum dot, the Kondo resonance separates into Zeeman states at a much lower magnetic field, $g \mu_{B} B_{c} \ll k_{B} T_{K}$, so that the Kondo temperature naïvely determined from $2 g \mu_{B} B_{c}$ is in disagreement with the previous estimates (see Table I). This observation can be understood as the combination of two related effects. First, a partially screened Kondo impurity is expected to be highly polarizable in a small magnetic field, which constitutes a relevant perturbation to the free spin fixed point in the renormalization group sense. Second, the logarithmic cusp of the Kondo peak at low bias makes the splitting more pronounced than for the Lorentzian shape typical of a Fermi liquid in the fully screened case. To put this surprising observation on firmer ground, we have performed NRG calculations for the local density of states in a magnetic field for the single-channel Kondo models with spins $S=1 / 2$ and $S=1$. This numerical solution allows us to confirm that the underscreened Kondo resonance starts to unveil its magnetic excitations for Zeeman energies as low as $k_{B} T_{K} / 16$ [see Figs. 3(d) and 3(e)]. A further interesting check on our analysis lies on the extracted Zeeman splitting as a function of magnetic field [Fig. 3(c)]. For odd (even) diamonds, we find that the dispersion of the magnetic states, rescaled to the corresponding Kondo temperature, clearly shows the presence (absence) of a threshold. Also remarkable is how these data for our two different samples quite naturally fall on top of each other, another signature of Kondo universality.

In conclusion, we have given comprehensive experimental evidence for the occurrence of the Nozières and Blandin underscreened Kondo effect in even charge molecular quantum dots. Our analysis, based on the stark differences with respect to regular transport properties of fully screened impurities, was strengthened by NRG calculations. An unexpected magnetic field sensitivity of partially 

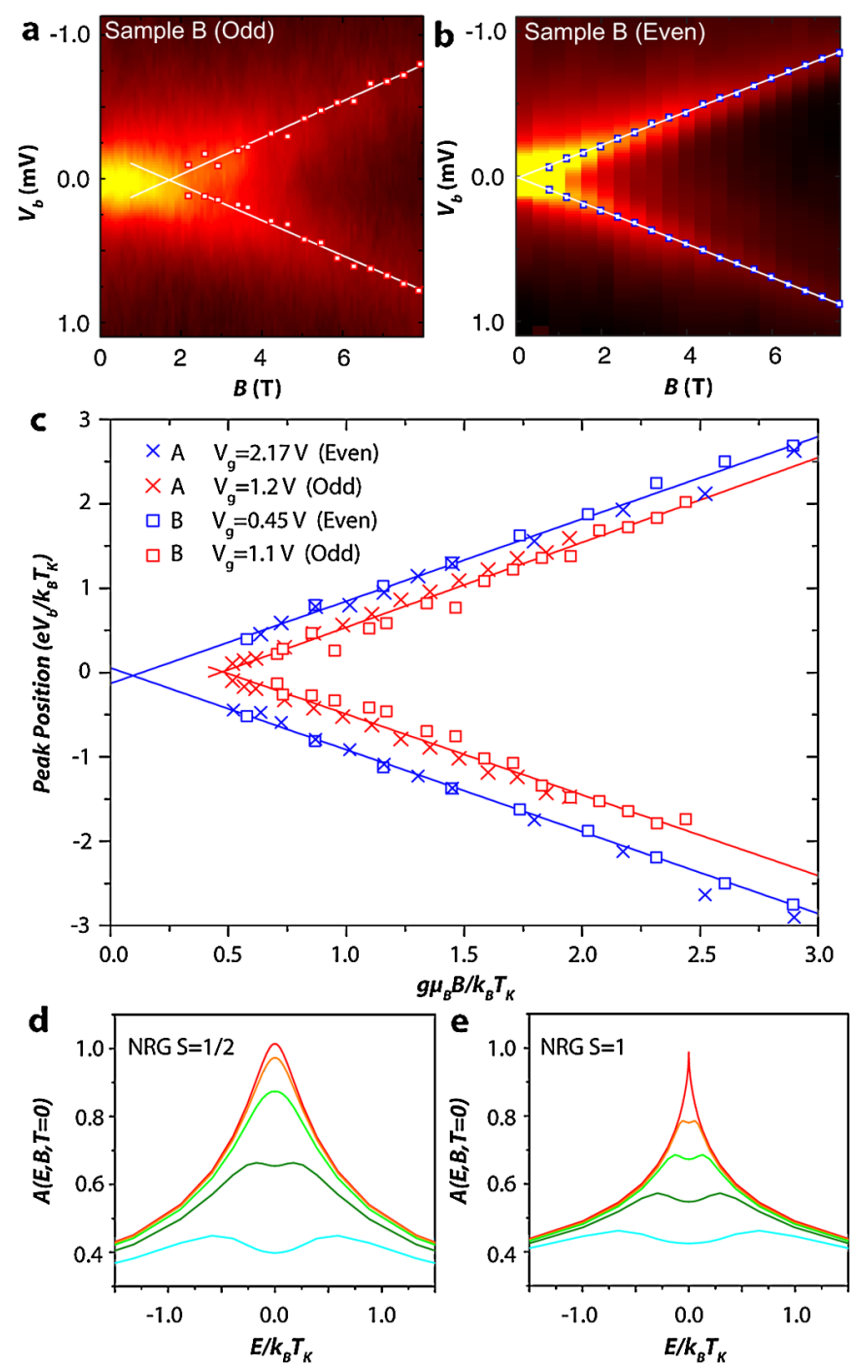

FIG. 3 (color online). (a), (b) Differential conductance versus bias voltage and magnetic field for sample $B$ in the odd and even diamonds (lines are linear fits to the $B$ dependence of the Zeeman peaks of sample $B$ ). (c) Positions of the Zeeman peaks of both samples and odd (even) diamonds as extracted from above. Linear fits provide the critical field $B_{c}$ by extrapolation to zero bias. Both bias voltage and magnetic field were renormalized by $T_{K}$ deduced from the HWHM at $B=0 \mathrm{~T}$ for each case. (d), (e) NRG calculations of the energy $E$ dependent equilibrium local density of states $A(E, B, T=0)$ at zero temperature, normalized to its $E=B=0$ value, for the spin $S=1 / 2$ and $S=1$ single-channel Kondo models and magnetic field values $g \mu_{B} B / k_{B} T_{K}=0,1 / 8,1 / 4,1 / 2$, and 1 (top to bottom).

screened Kondo impurities was also discovered that we could confirm theoretically. This work illustrates the striking versatility of molecular electronics for the investigation of fundamental aspects in quantum magnetism.

We thank E. Eyraud and D. Lepoittevin for their technical contributions, E. Bonet, T. Crozes, and T. Fournier for lithography development, and C. Winkelmann, C. Thirion, M. Desmukh, and L. Calvet for useful discussions. Samples were fabricated in the NANOFAB facility of the
Néel Institute. This work is partially financed by ANRPNANO Projects MolSpintronics No. ANR-06-NANO-27, MolNanoSpin No. ANR-08-NANO-002, ERC Advanced Grant MolNanoSpin No. 226558, and STEP MolSpinQIP.

[1] A. C. Hewson, The Kondo Problem to Heavy Fermions (Cambridge University Press, Cambridge, 1993).

[2] D. Goldhaber-Gordon et al., Nature (London) 391, 156 (1998).

[3] T. A. Costi et al., Phys. Rev. Lett. 102, 056802 (2009).

[4] P. Nozières and A. Blandin, J. Phys. (Paris) 41, 193 (1980).

[5] R. M. Potok, I. G. Rau, H. Shtrikman, Y. Oreg, and D. Goldhaber-Gordon, Nature (London) 446, 167 (2007).

[6] P. Coleman and C. Pépin, Phys. Rev. B 68, 220405 (2003).

[7] P. Mehta, N. Andrei, P. Coleman, L. Borda, and G. Zarand, Phys. Rev. B 72, 014430 (2005).

[8] H. Park, J. Park, A. K. L. Lim, E. H. Anderson, A. P. Alivisatos, and P.L. McEuen, Nature (London) 407, 57 (2000).

[9] J. Park et al., Nature (London) 417, 722 (2002).

[10] W. J. Liang, M. P. Shores, M. Bockrath, J. R. Long, and H. Park, Nature (London) 417, 725 (2002).

[11] L. H. Yu and D. Natelson, Nano Lett. 4, 79 (2004).

[12] J. J. Parks et al., Phys. Rev. Lett. 99, 026601 (2007).

[13] J. Schmid, J. Weis, K. Eberl, and K. V. Klitzing, Phys. Rev. Lett. 84, 5824 (2000).

[14] W. G. van der Wiel et al., Phys. Rev. Lett. 88, 126803 (2002).

[15] A. Kogan, G. Granger, M. A. Kastner, D. GoldhaberGordon, and H. Shtrikman, Phys. Rev. B 67, 113309 (2003).

[16] G. Granger, M. A. Kastner, I. Radu, M. P. Hanson, and A. C. Gossard, Phys. Rev. B 72, 165309 (2005).

[17] C. H. L. Quay et al., Phys. Rev. B 76, 245311 (2007).

[18] A. Posazhennikova and P. Coleman, Phys. Rev. Lett. 94, 036802 (2005).

[19] N. Roch, S. Florens, V. Bouchiat, W. Wernsdorfer, and F. Balestro, Nature (London) 453, 633 (2008).

[20] M. Pustilnik, L. I. Glazman, and W. Hofstetter, Phys. Rev. B 68, 161303 (2003).

[21] P. Roura-Bas and A. A. Aligia, Phys. Rev. B 80, 035308 (2009).

[22] N. Roch, S. Florens, V. Bouchiat, W. Wernsdorfer, and F. Balestro, J. Low Temp. Phys. 153, 350 (2008).

[23] F. Mallet et al., Phys. Rev. Lett. 97, 226804 (2006).

[24] See EPAPS Document No. E-PRLTAO-103-001945 for a description of temperature, bias, and magnetic field scaling. For more information on EPAPS, see http://www. aip.org/pubservs/epaps.html.

[25] M. Grobis et al., Phys. Rev. Lett. 100, 246601 (2008).

[26] G. D. Scott, Z. K. Keane, J. W. Ciszek, J. M. Tour, and D. Natelson, Phys. Rev. B 79, 165413 (2009).

[27] S.M. Cronenwett, T.H. Oosterkamp, and L.P. Kouwenhoven, Science 281, 540 (1998).

[28] A. Kogan et al., Phys. Rev. Lett. 93, 166602 (2004).

[29] T. A. Costi, Phys. Rev. Lett. 85, 1504 (2000). 